\title{
Extended Bandwidth Allocation in Cloud Computing
}

\author{
Ganesh Sherkar ${ }^{1}$, Suraj Pathak ${ }^{2}$, Dhananjay Newaskar ${ }^{3}$, Swapnil Bhakare ${ }^{4}$, A. P. Kulkarni ${ }^{5}$ \\ UG students, Sinhgad Institute of Technology, Lonavala, Maharashtra, India ${ }^{1,2,3,4}$ \\ Professor, Sinhgad Institute of Technology, Lonavala, Maharashtra, India ${ }^{5}$
}

\begin{abstract}
We have entered into a new era of computing, Enterprises are clearly moving from the "kicking the tires" stages to initial implementation of cloud computing and it's all about the "cloud" where Data sharing is an important functionality in cloud storage over the internet There is a great deal of interest in better leveraging the benefits of cloud computing as Cloud computing is a model which enables universal, convenient, on-demand network access to computing resources that can be rapidly accessed and released with minimal effort or service provider interaction. Capabilities in Cloud can be rapidly and elastically provisioned - in some cases automatically - to quickly scale out and rapidly released to quickly scale in. Bandwidth allocation plays a major role in sharing the resources towards the data center networks. Server performance is the major problem in cloud computing. When multiple users requests for the same server at a time, the performance of the server degrades. So we describe a innovative method of re-arranging the bandwidth dynamically from passive users to active users and vice versa as well as some users are allocated bandwidth statically using bandwidth mutual sharing and fair sharing technique.
\end{abstract}

Keyword: Performance analysis, Bandwidth mutual sharing, Fair sharing techniques.

\section{INTRODUCTION}

Cloud Computing is a emergent technology in today's to be done while maintaining high levels of reliability and world, Cloud computing is Internet-based computing, security.

where in resources, software and information are provided to computers and other devices on-demand, like the electricity grid

First, cloud computing is a broad term. It's as broad as saying "desktop computing" (i.e. the PC), which encompasses everything from the microchip to the Windows operating system to the software. As we will learn in this eBook, cloud computing encompasses all the same elements as the desktop

Second, you can't touch the cloud. Desktop computing is easy to understand because you can see, touch and feel your PC. The cloud is real, but it is abstracted to the point where you cannot see it, so it's harder to imagine.

The term "cloud computing", for a variety of very good reasons, has become very popular, and there are plenty of new and established IT companies that want to jump on the bandwagon, often incorrectly labeling anything to do with remote computing as the "cloud". cloud computing encompasses other forms of computing beyond software, including the core hardware (infrastructure) and platforms. In many ways, cloud computing is amazingly similar to desktop computing in that it encompasses the same three basic elements: hardware (infrastructure), OS (platforms), and software.

The main characteristics of cloud computing is dynamic provisioning. Dynamic provisioning allows the provision of services based on current demand requirements. This is done automatically using software automation, enabling the expansion and contraction of service capability, as needed. This dynamic scaling needs

(IaaS) Infrastructure-as-a-Service, IaaS - cloud providers supply the resources on-demand from their large pools installed in data centers. For wide-area connectivity, customers can use either the Internet or the carrier clouds .

(Pass) Platform-as-a-service, In the PaaS model, cloud providers bring a computing platform usually 
including OS, programming language execution environment, database, and web server .

(Sass) Software-as-a-service is a model wherein cloud providers install and operate application software in the cloud and cloud users access the software from cloud clients

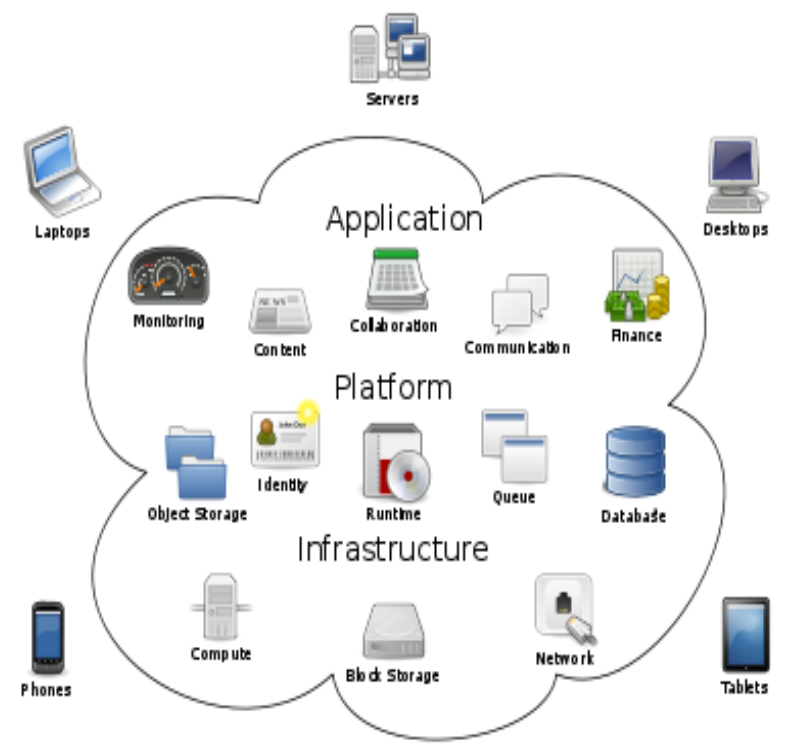

Fig 1: Cloud Users

Cloud computing provides convenient, on-demand network access to a shared pool of configurable computing resources is that could be Mobile Users or Users PC's. as shown in figure 1. Cloud Services are Classified into three Types IaaS, PaaS, SaaS. As shown in figure 2.

The main problems in cloud computing

1) Security and Privacy: Perhaps two of the more "hot button" issues surrounding cloud computing relate to storing and securing data, and monitoring the use of the cloud by the service providers.

2) Lack of Standards: Clouds have documented interfaces; however, no standards are associated with these, and thus it is unlikely that most clouds will be interoperable.

3) Continuously Evolving: Cloud Usesr requirements are continuously progressing, as are the requirements for interfaces, networking, and storage have increased gradually. This means that a "cloud" especially a public one, does not remain static and is also continuously evolving.

4) Static bandwidth allocation: Wastage of bandwidth so the server performance are decreased, maintenance are difficult.

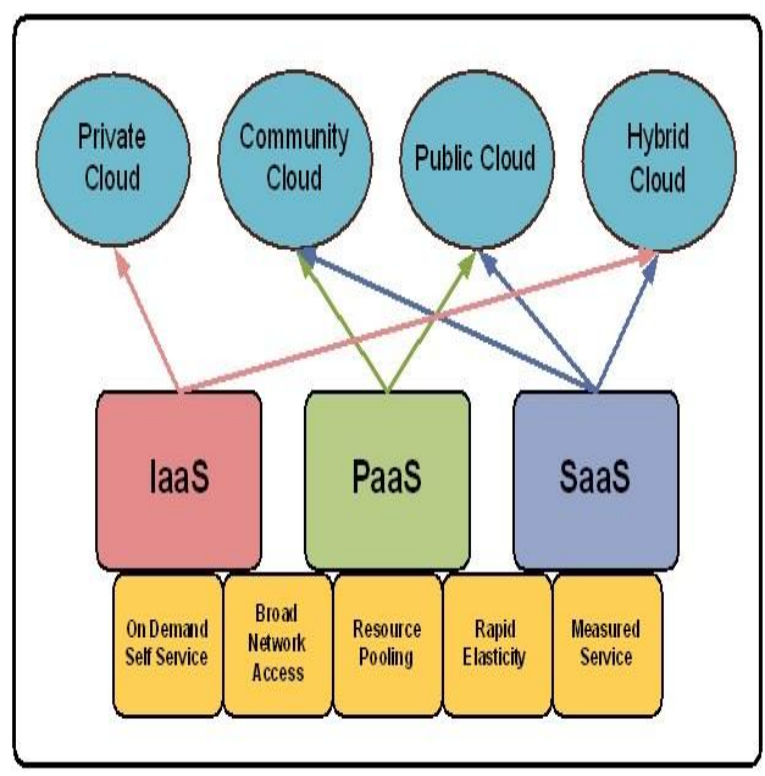

Fig 2: Cloud Services

\section{LITERATURE SURVEY}

The existing process focused on increasing the performance of the server by solving it to obtain accurate estimation of bandwidth distribution statically the complete probability distribution of the request and response time and other important performance indicators which has been described in novel approximate analytical model for performance evolution of cloud server .

The model allows cloud operators to determine the relationship between the number of servers and input buffer size that handles requests, on one side, and the performance indicators such as mean number of tasks in the system, blocking probability, and probability that a task will obtain immediate service, on the other services.

It is vital to isolate the network performance between the clients for ensuring fair usage of the constrained and shared network resources of the physical machine. Inappropriately, the existing network performance isolation techniques are not effective for cloud computing systems because they are difficult to adopted in a large scale and require non-trivial modification to the network stack of a guest OS.

\section{III.PROBLEM STATEMENT}

1) Bandwidth wastage and reduce performance of the server is the major Issue: The number server is moderately small, which makes them unfit for performance analysis of cloud computing data centers. The Users may vary and appear typically of the order of hundreds or thousands; traditional their request queuing analysis rarely considered systems of this size. Due to the diverse nature of the cloud environments, uniformity of user's requests and time dependency of load, cloud centers must provide expected quality of service at widely varying loads gradual increase of active users should not affect the performance of the system. 
2) More expensive: Cloud does not provide Passive users loses their cost as they are less likely to be active, They not even mention the priority in static bandwidth allocation. So the passive user lost their cost.

3) Performance: Unresponsive System is less likely to be sustainable A performance problem may be identified by slow or unresponsive systems. This usually occurs as the number of active users gradually increases, causing some part of the system to reach a limit in its ability to respond.

\section{PROPOSED SYSTEM}

Bandwidth Management is to be taken care of for gradually increasing the performance of the server, it is needed to re-allocate the bandwidth from passive user to active users and vice versa constantly as performance is the main issue customers are paying for. To perform the process of execution, the entire implementation is shared into four modules and those modules are as follows.

- Cloud server offers

- Performance- analysis queue

- Bandwidth allocation

- Bandwidth mutual sharing

1) Cloud Server Offers: Usually most of the users are started to access cloud due to the latest evolved technologies, for accessing cloud we require certain bandwidth resources which cloud has to offer us. Among all the resources bandwidth is the important one as performance is the main issue customers are paying for, if the availability of bandwidth is good then only the user can access the cloud well without any net traffic.

2) Performance- Analysis Queue: Bandwidth is managed from server side It is the technique to set up the preferred bandwidth in the server region. Computational accuracy and the precision of the final result have a complex, non-monotonic relation, so that in general an increase of precision can lead to a decrease of accuracy.

3) Bandwidth Allocation: When a user demanding for a resource to the cloud server which was being processed by multiple client then the users resource will be allocate to the cloud server according to the CPU usage of the cloud server which was verified by the main server. The cloud server should be designed in the way that it should response simultaneously for all the users under the performance analysis as well bandwidth allocation so We have to create a cloud server in the performance analysis way, which will do multiple resources simultaneously and if multiple users is trying to access the client at a time. The ideology behind this is to allocate and re-allocate the bandwidth if not used The mechanism is improved for the purpose of connectivity since it uses the concept of bands created by partitioning the client using multiple clients. These bands are used to help control the direction of data flow of server, the key idea is that our approach will reduce the propagation of energy thus reducing energy consumption.

4) Bandwidth Mutual Sharing: Here there is a way to check the speed and bandwidth allocated to the active users connected to the network have to see if others in the different routers have somehow prioritized their computers over mine, or have restricted the amount of bandwidth which is used by other users. Bandwidth is properly distributed to users where there are some users who are active for a long period while some are not active all the time so bandwidth is set statically to these users while the active users have dynamic allocation of bandwidth which is changed as number of users increases The fixed-size window control can achieve fair bandwidth sharing according to any of these criteria.

\section{CONCLUSION}

We proposed an innovative approaches for increasing the performance of the server in cloud computing. Reallocating of bandwidth from passive user to active user using the technique bandwidth-mutual sharing and fair sharing techniques, and static bandwidth is allocated to Users who are active from long time while new Users are allocated bandwidth Dynamically. As well as users log is maintained which helps to allocate bandwidth as per users use. The future scope for this would be when the number of client's increase to maximum level then the load would be divided among two or more Server.

\section{REFERENCES}

[1] International Journal of Research in Advent Technology, Vol.2, No.12, December 2014 E-ISSN: 2321-9637

[2] IRACST - International Journal of Computer Networks and Wireless Communications (IJCNWC), ISSN: 2250-3501 Vol.3, No2, April 2013

[3] International Journal of Science, Engineering and Technology Research (IJSETR) Volume 2, Issue 1, ISSN: 2278 - 7798 January 2013

[4] 2013 International Conference on Intelligent Systems and Signal Processing (ISSP)

[5] [IJESAT] International Journal OF Engineerig Science \& Advanced Technology Volume-2, Issue-5, 1372 - 1376 ISSN: 2250-3676

[6] 2013 International Conference on Intelligent Systems and Signal Processing (ISSP) 